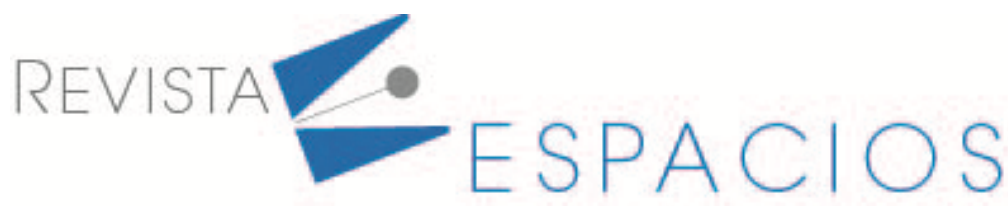

\title{
Resiliencia de los sistemas socio naturales ante el cambio climático de los pueblos originarios Weenhayek en Bolivia
}

\section{Resilience of socio-natural systems to climate change in the Weenhayek indigenous peoples in Bolivia}

ROJAS, Juan M. ${ }^{1}$

ZÚÑIGA, Libys M. ${ }^{2}$

\section{Resumen}

Los pueblos originarios en Bolivia han dejado de cumplir con su función de adaptabilidad socio ambiental. A través de elementos teóricos y el análisis de relación conceptual de la adaptación y resiliencia, apoyados en concepciones interdisciplinares desde un enfoque sistémico, se plantea tratar la adaptación planificada como componente de la resiliencia como constribución de mejora de los sistemas socio ambientales y las construcciones tradicionales, siendo este el objetivo del presente artículo.

Palabras clave: resiliencia, adaptación planificada, sistemas construidos, pueblos originarios.

\begin{abstract}
The native peoples in Bolivia have stopped fulfilling their function of socio-environmental adaptability. Through theoretical elements and the analysis of the conceptual relationship of adaptation and resilience, supported by interdisciplinary conceptions from a systemic approach, it is proposed to treat planned adaptation as a component of resilience as a contribution to the improvement of socioenvironmental systems and traditional constructions. this being the objective of this article.

keywords: resilience, planned adaptation, built systems, indigenous peoples.
\end{abstract}

\section{Introducción}

Los pueblos indígenas no son una parte romántica de un pasado terminado, ni una curiosidad de museo de un presente globalizado. Son sociedades que mantienen una fuerte vitalidad, que conservan sus particularidades tradicionales adaptándolas a los nuevos tiempos, Barabas, A. (2014). Bolivia ha sido y es un país mayoritariamente indígena pero internamente muy diverso. Se representan en un alto número pequeños pueblos indígenas (guaraní, Chiquitano, Mojeño, Weenhayek, entre otros) en el territorio nacional. De esta manera los pueblos originarios, adaptan sus usos y costumbres a la nueva realidad multicultural y multiétnica, ocupando en muchos casos territorios ajenos y deconocidos a ellos. Barabas, A. (2014).

En Bolivia los Weenhayek se establecieron en una estrecha franja del Sureste del departamento de Tarija, provincia del Gran Chaco; en las comunidades de Ibibobo, Crevaux, D`orbigny, entre otras, en frontera con la

${ }^{1}$ Consultor externo. Departamento de Obras Civiles. Doctorando de la Universidad Internacional Iberoamericana, México. mark_3214@hotmail.com 2Docente .Departamento de Construcciones . Universidad de Holguín, Cuba. Correo electrónico de contacto Imzi@uho.edu.cu 
República Argentina. Viven en aldeas de diferentes tamaños, que comprenden de 1 a 100 familias extendidas. La distribución espacial del pueblo refleja las relaciones sociales, que por lo general son lazos familiares entre todos sus habitantes. Un "asentamiento Wichí o Weenhayek" (wichiwetes) es una suerte de comunidad residencial y familiar, además de ser en términos materiales un conjunto de "moradas": viviendas, rasgos del paisaje y edificios de uso comunitario.

En referencia a las construcciones Weenhayek, estas se han ido modificando en cuanto al empleo de materiales y técnicas constructivas, a causa de factores varios como: la restricción de acceso a los recursos naturales, la destrucción de su medio ambiente, la ganadería extensiva, el aumento de la pobreza, el desplazamiento de sus terrenos, entre otros. En referencia a las técnicas constructivas y materiales de construcción empleados, se caracterizan por el empleo de sistemas constructivos húmedos (tierra) en las regiones que presenta cuerpos de agua superficiales y sistemas constructivos secos (madera) en las regiones de difícil accesibilidad al agua. Estas construcciones son vulnerables ante la ocurrencia de desastres relacionados al clima se viene incrementando desde la década del noventa.

Las afectaciones climáticas de origen natural asociados con el régimen de lluviano solo comprometen su hábitat, sino que es el responsable del abandono de sus tradiciones, hogares y terrenos (debido a que ya no cumplen con la función de protegerlos de las inclemencias climáticas). Este abandono ocasiona que los Weenhayek ocupen construcciones muchas veces improvisadas compuestas de ladrillos, hules, materiales contemporáneos, entre otros, producto de programas de viviendas sociales o viviendas espontáneas durante los distintos periodos de afectaciones climáticas. Entre las principales afectaciones a sus viviendas se tiene las inundaciones, que afectan a numerosas familias debido al desborde del río Pilcomayo, el cual se produce por la saturación de sedimentos en el fondo del mismo producto de materiales arrastrados desde la serranía del rio Aguarague causados por las fuertes lluvias aguas abajo. Es por ello que existe la necesidad de rescatar, preservar y mejorar el hábitat de los pueblos originarios como el Weenhayek en la región del Gran Chaco ante los riesgos de origen climático, adaptando estas construcciones a la nueva realidad social, económica y ambiental buscando la preservación de sus rasgos culturales.

Desde dicha perspectiva, la Oficina del Sistema de las Naciones Unidas para la Reducción del Riesgo de Desastres ([UNISDR], 2009), reconoce variables que intervienen en el análisis de los riesgos como las amenazas y la vulnerabilidad. En la presente investigación, las amenazas antrópicas, son las provocadas por las actividades humanas como la agricultura, la ganadería, el transporte, la industria, entre otras, que han demostrado que han afectado todo el sistema climático como evidencia de la influencia humana en el sistema climático, Intergovernmental Panel on Climate Change, ([IPCC], 2014)].El otro componente del riesgo es la vulnerabilidad, la cual el ([IPCC], 2014a), la define como la propensión o predisposición a ser afectado negativamente, comprendiendo una variedad de conceptos y elementos que incluyen la sensibilidad o susceptibilidad al daño y la falta de capacidad de respuesta y adaptación. También se refiere al,

nivel de exposición que presenta determinado grupo humano frente a las amenazas en su medio. Es la debilidad de una comunidad para resistir los efectos de una amenaza y para recuperarse después de ésta. Una población es vulnerable cuando se interrelacionan factores de tipo cultural (forma de reaccionar frente al riesgo), ideológico (imagen o concepción del mundo, en especial frente a los desastres), educativo (la forma en que una comunidad se informa y se prepara frente al riesgo), político (existencia o no de regulaciones legales encaminadas a mitigar los efectos del riesgo), técnico (presencia y estado de diseños y estructuras adecuadas para afrontar situaciones de riesgo), entre otros, (Baró, J. y Monroy, F., 2018, p.20).

La vulnerabilidad, tiene componentes que la condicionan y depende de la exposición, la susceptibilidad y de la resiliencia. De ahí que autores (Narváez, L. et. al., 2009; Rojas, O. y Martínez C., 2011; Vargas J. y Paneque, P., 
2016; Baró, J. y Monroy, F., 2018; [IPCC], 2014b), conceptualizan la exposición al riesgo a la población, las propiedades, medios de vida, sistemas y otros elementos presentes en las zonas que pueden verse afectados por amenazas. Es la condición de desventaja, debido a la ubicación, posición o localización de un sujeto, objeto o sistema expuesto al riesgo, (UNISDR, 2009).Chacón, J. (2008), define a la susceptibilidad, como aquella que se basa en la delimitación de la distribución espacial de factores relacionados con los procesos de inestabilidad, sin que se derive implicación temporal alguna. También es conceptualizada por otros autores como la mayor o menor predisposición a que un evento suceda u ocurra sobre determinado espacio geográfico, (Centro Internacional para la Investigación del Fenómeno de El Niño [CIIFEN], 2010; Ministerio de Ambiente y Desarrollo Sostenible de Colombia [MADSC], 2014 y Soldano, A., 2015). Mientras la ([UNISDR], 2009), la define como el grado de fragilidad interna de un sujeto, objeto o sistema para enfrentar una amenaza y recibir un posible impacto, debido a la ocurrencia de un evento adverso, aspecto en los que se coincide.

El último componente de la vulnerabilidad es la resiliencia. La palabra resiliencia tiene su origen en el latín resilio(Romero, O. et. al., 2017), que significa volver atrás, volver de un salto, resaltar, rebotar. En cuanto a la resiliencia la Real Academia Española ([RAE], 2020a), la define como la capacidad de un material, mecanismo o sistema para recuperar su estado inicial cuando ha cesado la perturbación a la que había estado sometido.

El concepto de resiliencia no es uniforme y depende de quienes la usan, del contexto en que se utiliza, y del objeto y de la escala de análisis, Rebotier, J., et. al.(2013). En la actualidad este término está en el debate desde varias disciplinas por las particularidades de dicho término: flexible y adaptable a las diferentes perturbaciones que pueda tener el sistema de análisis: natural, social(Ojeda, D. y Steffens, E., 2017; Atencia, D., et. al., 2020), económico, político, tecnológico, entre otros, (Zúñiga I., 2017). La resiliencia para los sistemas ecológicos fue descrita por primera vez en el libro Resilience and stability of ecological systems de Crawford Stanley Holling, en 1973y la consideró como: "la medida de la estabilidad de tales sistemas para absorber cambios e incluso persistir [...], un sistema puede ser bastante resiliente, fluctuar demasiado y tener una baja estabilidad," (Holling, S. 1973, p. 17).En ese sentido, (UNISDR, 2009), la define como la capacidad de un sistema, comunidad o sociedad expuestos a una amenaza para resistir, absorber, adaptarse y recuperarse de sus efectos de manera oportuna y eficaz, lo que incluye la preservación y la restauración de sus estructuras y funciones básicas.

Desde estas concepciones generales para mantener capacidades resilientes en cualquier tipo de sistema y manifestar algunas formas de estabilidad (Zúñiga I., 2017), hay que controlar la resiliencia. Es por ello que resulta tan importante el análisis de la resiliencia porque:

si las amenazas son conocidas y se trabaja sobre la reducción de la exposición y la susceptibilidad desde una concepción de la preparación de los sistemas construidos y sociales planificadamente, a través del fortalecimiento de la resiliencia de los diversos componentes expuestos y susceptibles de los sistemas urbanos y territoriales, entonces se puede considerar que a mayor resiliencia menor es el riesgo, (Zúñiga I., 2018, 43).

En revisión de la literatura científica sobre la capacidad de adaptación como componente de la resiliencia es un tema poco tratado en la literatura consultada hasta la etapa por este autor, (Programa de las Naciones Unidad para el desarrollo [PNUD], 2011; Picket, S. et. al., 2014; Lara, R. y Vides-Almonacid, R., 2014; Magrin, G., 2015 y Sepúlveda, J., 2015).Como campo de investigación se trata a la resiliencia en la que se reconocen teorías, conceptos, dimensiones, enfoques, atributos, variables, indicadores e índices y otros desarrollos teóricos conceptuales que han ayudado sustancialmente a entenderla, fundamentalmente desde enfoques integrados a otras disciplinas. Sin embargo la resiliencia desde el papel de su capacidad de adaptación para los sistemas socio naturales ante el cambio climático ha sido limitado. Es por ello que el objetivo del presente artículo es esclarecer el papel de la capacidad de adaptación en la resiliencia para los sistemas socio naturales ante el cambio climático 
en el orden teorico conceptual que ayude al entendimento de estos procesos para su aplicación en contribución a la mejora del habitat en los pueblos originarios Weenhayek en Bolivia.

\section{Metodología}

El desarrollo de la investigación se realizó a través de métodos teóricos (histórico, lógico, inducción, deducción, revisión de la bibliografía) y del análisis cualitativo de carácter descriptivo, explicativo y evaluativo. Dichos métodos han sido apoyados por las concepciones interdisciplinares desde un enfoque sistémico para tratar la resiliencia en los sistemas socio naturales ante el cambio climático en los pueblos originarios desde un enfoque de la adaptación planificada ante los efectos del cambio climático.

El análisis se realizó en materia conceptual sobre la resiliencia en los sistemas socio naturales, para lo cuál se estudiaron un total de 39 autores ${ }^{1}$ desde un marco teoricoconceptual. Para ello se utiliz ó el procesador Ucinet for Windows: Software for Social Network Analysis, [UCINET], versión 6.710, (Borgatti, S., et. al., 2002), y a través de matrices binarias para el tratamiento de un grupo de variables como cualidad, función y finalidad que son recurrentes y califican la resiliencia por los autores referidos con anterioridad. Este análisis develó la centralidad entre ellas, lo que permitió un desarrollo conceptual que ayuda a entender el cómo trabajar la resiliencia de los sistemas socio naturales ante el cambio climático como forma de adaptación planificada en los pueblos originarios bolivianos.

\section{Resultados}

Entender la resiliencia como una herramienta de flexibilidad en rangos y parámetros es importante, porque depende de cada configuración territorial, de procesos básicos externos, así como de las amenazas de cualquier región y del lugarcomo un aspecto esencial que brinda enfoques de sostenibilidad. Para ello, en el proceso de análisis del desarrollo conceptual de la resiliencia, se establecen tres matrices binarias para determinar cualidades, funciones y finalidad del término resiliencia cuando se utiliza en el marco socio natural ante el cambio climático. Para ello los resultado están como siguen:

\subsection{Matriz binaria con las "cualidades" de la resiliencia de los sistemas socio naturales ante el cambio climático}

Las variables identificadas como cualidades de la resiliencia no se correlacionan, pero si se agrupan en grupo de autores que utilizan determinados términos en una centralidad de acuerdo la cualidad de la resiliencia, la de: "capacidad"; ya sea de un sistema, colectividad, comunidad o sociedad expuestos a una amenaza con un nivel de correlación de 0.846 y un mayor poder en la red, lo que corrobora en la práctica general, la utilización de este término para calificar la resiliencia. También en menor medida se identifica como "capacidad de adaptación" (PNUD, 2011; Picket, S. et. al., 2014; Lara, R. y Vides-Almonacid, R., 2014; Magrin, G., 2015 y Sepúlveda, J., 2015) y finalmente un único autor, Clerc, J. y Díaz, M., (2016), la destaca como "adaptación".

${ }^{1}$ (Stanley, C., 1973; Grupo de las Naciones Unidas para el Desarrollo [UNDG], 2009; Bahadur, A. et. al., 2010; Uriarte, J., 2010; Leichenko, R., 2011; Programa de las Naciones Unidas para el Desarrollo [PNUD], 2011; Doring, T. et. al., 2013; Jiménez, M., 2013; Camarero, L. y Del Pino, J., 2014; Picket, S. et. al., 2014; Skerk, C., et. al., 2014; Vides-Almonacid, R., 2014; Altieri, M. et. al., 2015; Ávila, B. y Gonzáles, G., 2015; Kenneth, A., et. al. 2015; Magrin, G., 2015; Papa, R. et. al., 2015; Sepúlveda, J., 2015; Clerc, J. y Díaz, M., 2016; Del cura, F. y Quintero, E. ,2016; Fisichelli, N. et. al., 2016; Levinea, N., et. al., 2016; Peredo, S. y Vela, M., 2016; Pradilla, G., 2016; Quinlan, A. et. al., 2016; Sánchez, P. et. al., 2016; Shaikh, K. y Saidul, M., 2016; UN-Habitat, 2016; González, G. y Maldonado, L., 2017; Quevedo, A., 2017; Rivero, E., 2017; Timpane, B. et. al., 2017; Baró, J. y Monroy, F., 2018; Rodríguez, Y., 2018; Gonzáles, E., et. al. 2019; Guarino, G. y Pironto, A., 2019; Hardy, V., et. al., 2019; Aguilar, L., 2020; Sandoval, J., 2020) 
La referencia de la variable "capacidad" al destacarse como la de mayor centralidad dentro de las cualidades de la resiliencia se analiza como complemento desde la generalidad de los sistemas. Sin embargo esta investigación considera pertinente que aunque ha tenido un valor de 0.128 , la "capacidad de adaptación" a modo de complemento directo de esa capacidad califica en términos de interdependencias a los cuales los sistemas deben tener como propiedad intrínseca, como se muestra en la figura 1.

Figura 1

Cualidades de la resiliencia según autores

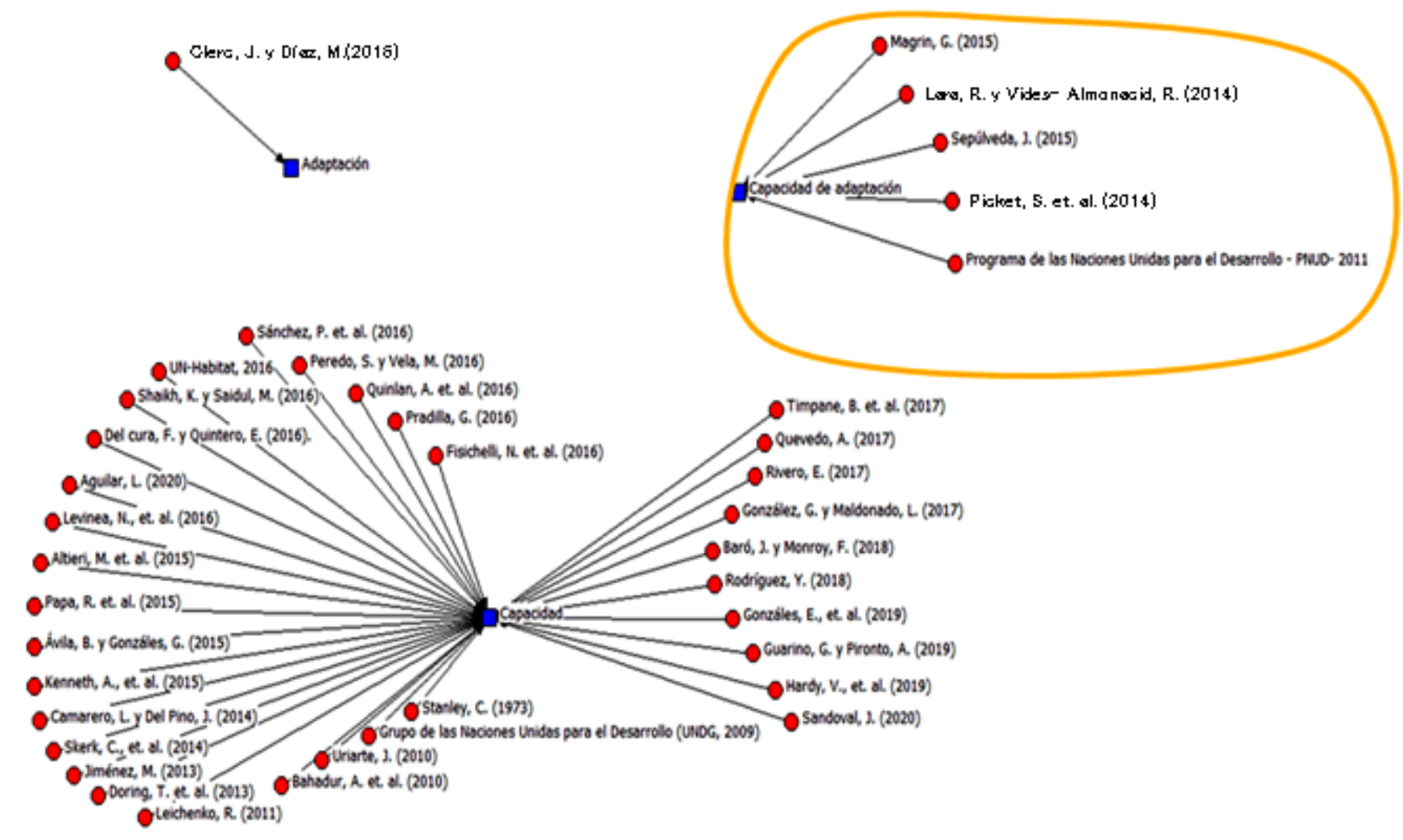

Fuente: elaboración propia

Desde esa perspectiva, la Real Academia Española ([RAE], 2019), define a la adaptación como la acción de acomodar, es decir hacer que una cosa se ajuste a otra. Lara, R. y Vides-Almonacid, R., (2014) la consideran como el ajuste de un organismo, y como la comprensión y transformación del entorno cambiante a través de la cultura. En los sistemas naturales o humanos se asocia con la reducción y el manejo de los riesgos relacionados con el cambio climático, Organización de las Naciones Unidas ([ONU], 2015).

Por otro lado, la adaptación se estructura en dos grupos: la adaptación autónoma y la planificada, (ONU, 2015). En el primer caso, esta misma organización mundial la define como los ajustes realizados por los ecosistemas y los humanos sin intervención externa en respuesta a un entorno cambiante, donde todos los sistemas (sociales y ecológicos) tienen cierta capacidad de adaptación autónoma. En cuanto a la adaptación planificada, es una intervención humana deliberada con la intención de aumentar la capacidad del sistema para sobrevivir y funcionar en un nivel aceptable bajo las condiciones climáticas de un sitio específico, (ONU, 2015).Entender la capacidad de adaptación como una función de la resiliencia es un tema poco tratado en la literatura consultada hasta la etapa por este autor, (PNUD, 2011,Picket, S. et. al., 2014; Lara, R. y Vides-Almonacid, R., 2014; Magrin, G., 2015 y Sepúlveda, J., 2015) que ayuda a esclarecer el papel de la adaptación. 


\subsection{Matriz binaria con las "funciones" de la resiliencia de los sistemas socio naturales ante el cambio climático}

En el proceso de análisis del desarrollo conceptual de la resiliencia a través de las variables que determinanlas funciones de la "capacidad". La RAE, (2020b), define al vocablo "función" como la capacidad de actuar propia de los seres vivos y de sus órganos; y de las máquinas o instrumentos. De ahí que el conocer las funciones características que califican la capacidad de adaptación de la resiliencia permite comprender la interactuación e interrelación existente entre adaptación, capacidad y resiliencia desde dicha perspectiva.

La matriz binaria permitió establecer una red que devela la centralidad de las funciones de la resiliencia, que se muestra de color azul en la figura2, en la cual "absorber", "resistir", y "recuperar", muestran la mayor correlación de autores analizados con centralidades de $0.455,0.333$ y 0.333 respectivamente y por otra, los mayores grados de intermediación es decir, la posibilidad que tienen estas funciones para intermediar las comunicaciones con las otras variables a ellas conectadas.

De ahí que se determinan como las tres funciones principales de la resiliencia en la presente investigación, donde se corrobora la importancia de estas funciones:

1. "absorber"; o consumir enteramente algo, permite convertir, transformar y (o) asimilar las alteraciones u otros fenómenos externos como parte suya, haciéndolos inherentes a él (RAE, 2020c);

2. "resistir"; o el oponerse a la acción o violencia de otra, RAE (2020d), permite a un todo, un sistema o un ser defenderse, tolerar, condescender y (o) soportar a manera de hacer prevalecer sus rasgos y (o) características ante acciones externas e internas; $y$

3. "recuperar", definido como el volver a tomar o adquirir lo que antes se tenía, (RAE, 2020e), permite tornar un sistema o unidad a su estado previo y (o) antes de una variación producto de una amenaza externa o interna. Para lo cual debe de desarrollar estrategias, tácticas, acciones y técnicas que permitan corregir, mitigar, y compensar los impactos y efectos ocasionados, permitiendo recuperar características propias.

Estas nociones manifiestan la existencia de un desarrollo interno dentro de la resiliencia, que genera continuamente un ciclo de gestión desde una secuencia de actividades, donde continuamente absorbe, resiste y recupera sus características, atributos, cualidades y funciones ante una acción externa a manera de conservar sus propiedades y volver a su estado de equilibrio, donde prevalecen su propiedades intrínseca.

El considerar la resiliencia como una capacidad de adaptación, que desarrolla distintas funciones como la absorción, resistencia y recuperación, requiere de una finalidad o el para qué. 
Figura 2

Funciones de la cualidad de la resiliencia

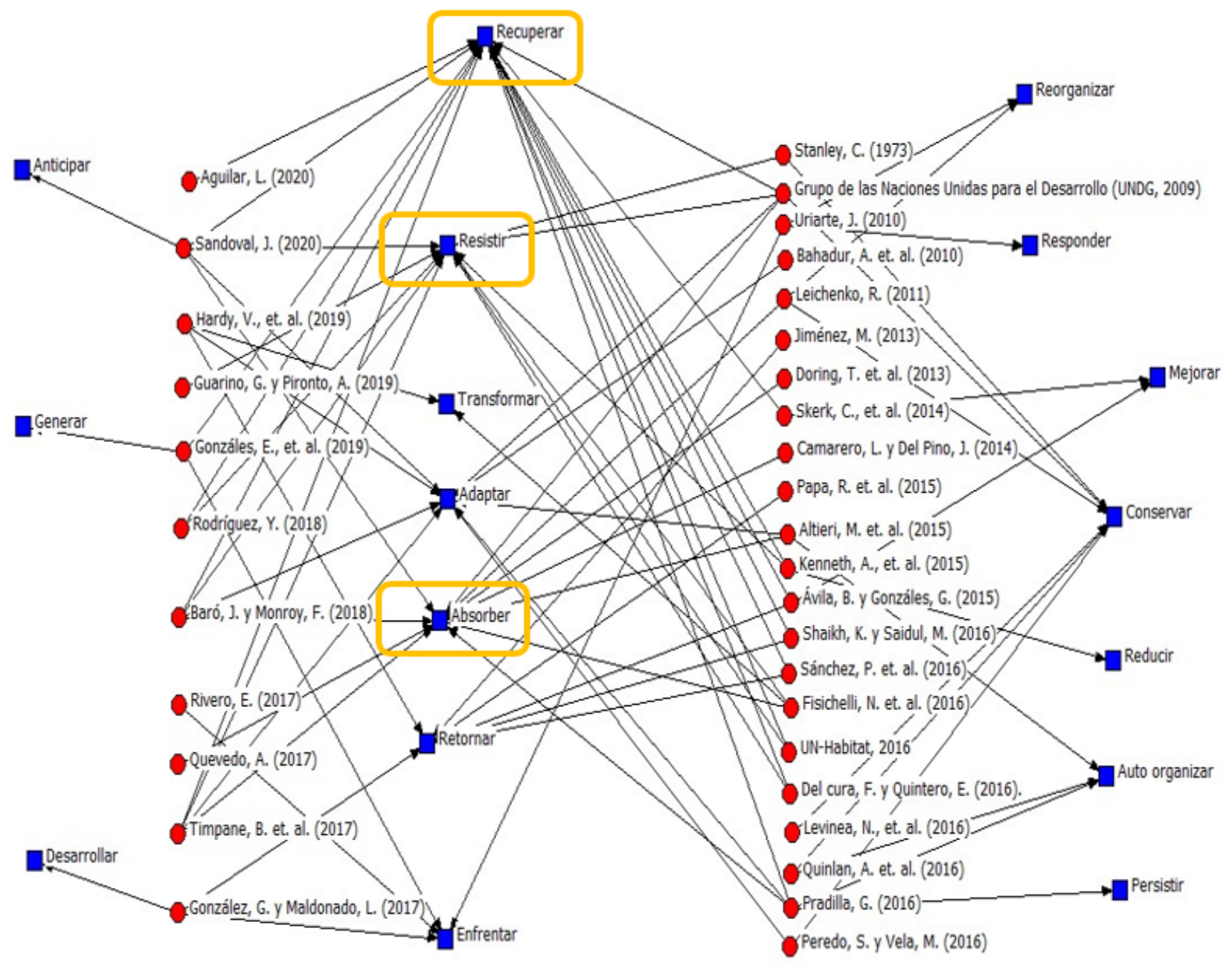

Fuente: elaboración propia

\subsection{Matriz binaria con la "finalidad" de la utilización de la resiliencia para los sistemas socio naturales ante el cambio climático}

Desde esa perspectiva, se organiza una tercera matriz binaria para determinar a través del consenso de autores la "finalidad" que califica la resiliencia mediante los distintos autores que se unen a un concepto común entre ellos, que se refleja en la figura 3. La "preservación del sistema" se determina como la finalidad más común para la resiliencia, con una centralidad de 0.394 .

La RAE (2020f), señala la preservación, como la acción o efecto de preservar, es decir, de proteger, resguardar anticipadamente a alguien o algo de algún daño o peligro. Este análisis establece que la finalidad busca proteger, amparar y (o) cuidar las características, propiedades y funciones del sistema. Además de precautelar el funcionamiento y normal desarrollo sin que exista alteración alguna, a manera de conservar cualidades, propiedades y (o) características ante un efecto adverso externo. 
Figura 3

Finalidad de la capacidad

resiliente según autores

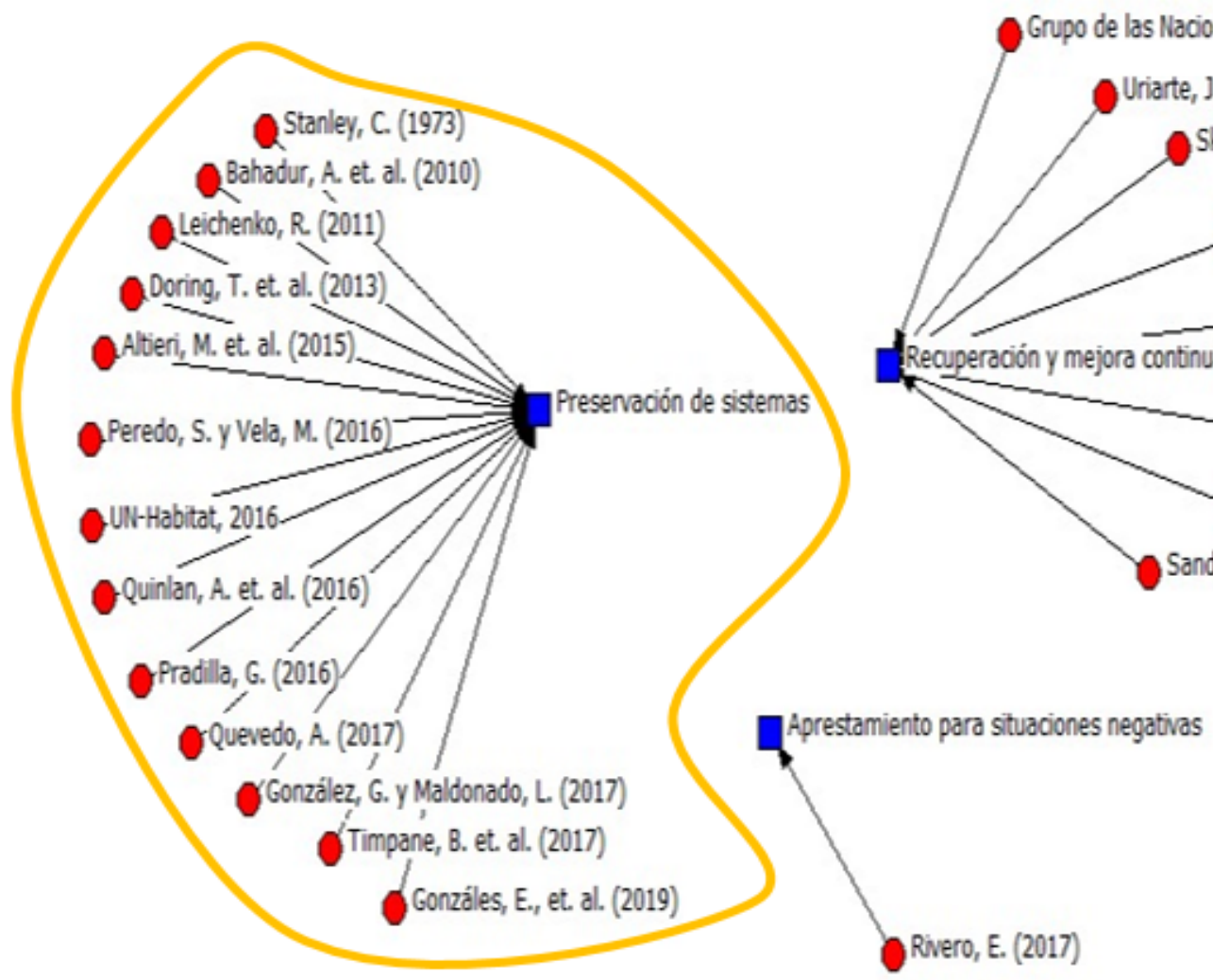

Aguilar, L. (2020)

Guarino, G. y Pironto, A. (2019)

Camarero, L. y Del Pino, J. (2014)
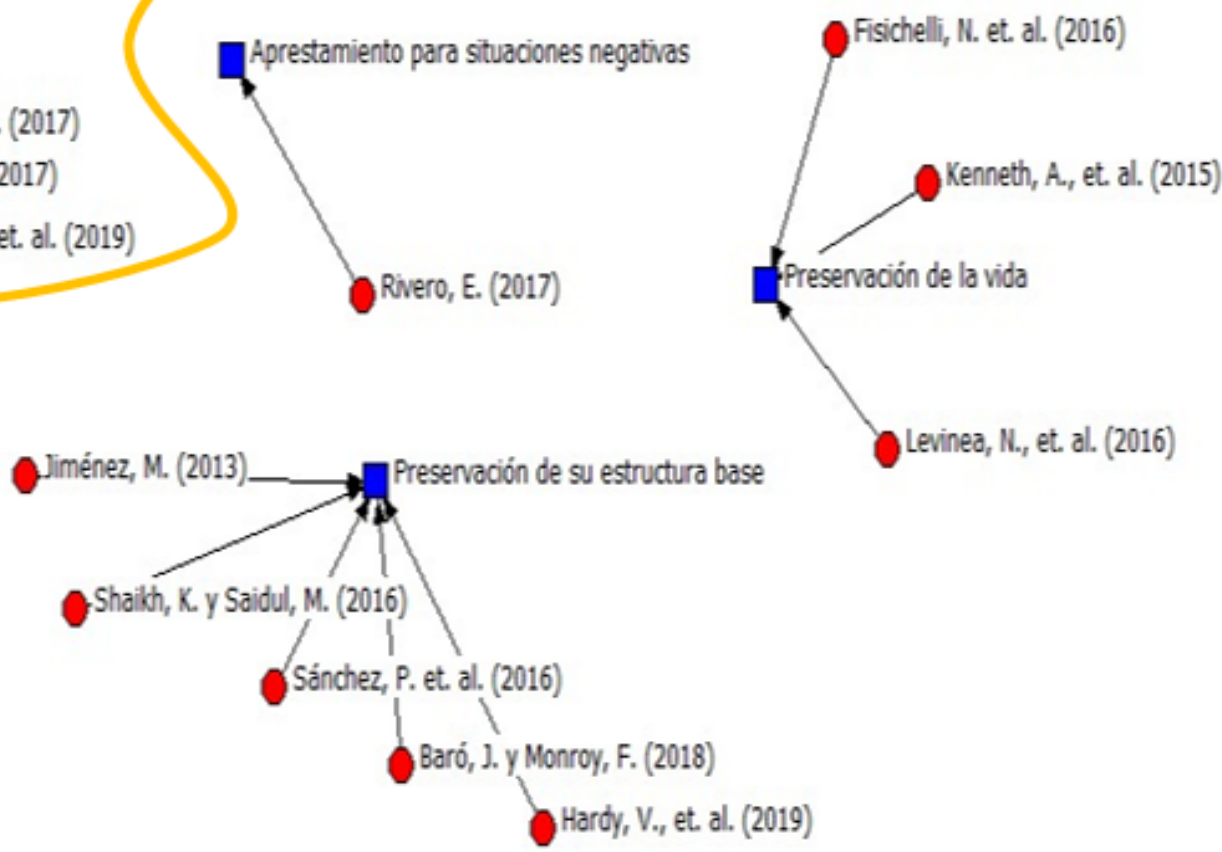

Fuente: elaboración propia

A modo de resumen, la resiliencia de los sistemas socio naturales ante el cambio climático es una capacidad de adaptación planificada que absorbe, resiste y se recupera para su preservación porque puede ayudar a contrarrestar y (o) afrontar los efectos del cambio climático puesto que intrínsecamente se muestra como coadyuvante a la mejora de la estabilidad de la condición humana en regiones que son amenazadas por determinados fenómenos extremos de origen climático.

Se destaca además que esa adaptación debe ser necesariamente planificada a través de la planificación territorial como ejercicio de anticipación para reducir riesgos y, a la vez, promover bienestar; no es solamente un proceso para minimizar la vulnerabilidad, sino también para identificar formas de maximizar oportunidades, Barton, J. (2009). Desde la adaptación planificada en la planificación territorial se demandan los mayores esfuerzos para 
mejorar los sistemas que intervienen como parte esencial del desarrollo de la vida de los seres humanos. En ese sentido,

debe concebirse como un proceso transversal que reduzca y reoriente las inequidades sociales, económicas y ambientales a través de la estabilidad integral e integrada de los sistemas naturales, construidos, y sociales para lograr riesgos aceptables de acuerdo a los niveles de desarrollo de las localidades, el aseguramiento de la permanencia, la calidad, el desarrollo de la vida de los seres humanos y el entorno que les rodea, (Zúñiga I, 2017, 45)

En la concepción anterior, esta misma autora, reconoce que son complementos necesarios que integran el resultado dialéctico que se produce entre la sociedad y la naturaleza, por lo que la adaptación planificada se desarrolla desde dos perspectivas:

la físico-espacial se considera como una capacidad de los recursos naturales y construidos para la absorción, resistencia y recuperación ante impactos, niveles de uso o límites establecidos por cada uno de ellos, que permiten mantener su autorregulación con niveles aceptados de conservación y estabilidad; $y$

la socio-política que se concibe como una capacidad de respuestas a partir de la conformación de valores organizados y estructurados en sistemas de significación, a través de la educación y la cultura del riesgo a desastres, (Zúñiga I, 2017, 47)

Esta investigación desde su desarrollo teórico deriva y completa la concepción del término capacidad de adaptación planificada en la resiliencia de los sistemas socio naturales ante el cambio climático al incorporar las perspectivas físico espaciales y socio políticas. En cuanto a la primera perspectiva, se reconocen las contribuciones desde el espacio y (o) localización, por una parte, y las capacidades físicas de sistemas construidos, por otra.

La condición de que espacio y lugar representan una forma de configuración territorial constituyen la materialidad y la sociabilidad del individuo y su sociedad ante los diversos componentes que limitan los medios de vida, a través de una relación sistémica del lugar donde se vive, en donde se vive, y cómo se vive. Esta se ve alterada en diversos lugares por los cambios que el mundo natural le prepara a las sociedades. Desde esas perspectivas, el saber construido y el saber nuevo, como formas de creación y vida, son eslabones esenciales para atenuarlas como forma de acción ante estas condiciones de la subsistencia humana.

En cuanto al funcionamiento a nivel urbano-territorial, constituye una expresión concreta de las formas de organización social y política que han determinado la ocupación, la posesión y la apropiación de los recursos, (Mateo, 2004) desde una consideración de la estabilidad socioambiental. Para ello se demanda ante todo de la evaluación del modelo espacial como reflejo del espacio físico, la distribución e interrelaciones entre los diferentes tipos de uso del suelo, con los objetos económicos y sociales; y los flujos y nexos que se establecen entre ellos desde la individualidad de cada uno.

Los sistemas construidos son inherentes al espacio, y son todas aquellas construcciones que sirven al ser humano para su hábitat, como las viviendas, las calles y las infraestructuras asociadas, las fábricas, espacios recreativos entre otras. En ese sentido, la resiliencia considera los elementos estructurales es decir todo aquello que conforma y determina una construcción, y es reconocida como capacidad de carga, en la cual,

Para los sistemas construidos, la capacidad de carga o de uso es dada por el ser humano en el diseño de los elementos que lo conforman y es otorgada por la tolerancia que cada elemento desarrolle en pos de su estabilidad de acuerdo al uso que se le dará a la edificación a diseñar. En ellas intervienen además las 
propiedades físicas y mecánicas de los materiales y los esfuerzos a los que se someten los materiales y elementos. Entre las propiedades físicas de los materiales se encuentran los análisis de la porosidad, compacidad, permeabilidad, capilaridad, heladicidad, higroscopicidad, conductividad térmica y conductividad eléctrica. En cuanto a las propiedades mecánicas de los materiales se analizan: la fuerza de rotura, tensión de rotura, tenacidad, dureza, fragilidad, elasticidad, plasticidad, rigidez, fatiga y fluencia. También es vital analizar los esfuerzos a los que se someten los materiales y elementos como la tracción, compresión, pandeo, cortadura o cizalladura, torsión y flexión, (Zúñiga I., et., al, 2020, p. 54).

La perspectiva socio-política se evidencia desde la construcción social de los espacios habitables del ser humano, donde se desarrolla desde un proceso normado y político el desarrollo socio-económico en el cual interviene la educación y la cultura como eslabones clave del desarrollo socio económico de cualquier territorio.

\section{Conclusiones}

La significación de la resiliencia de los sistemas socio naturales ante el cambio climático es una capacidad de adaptación planificada para la organización urbano territorial donde intervienen perspectivas físico espaciales y socio políticas de cualidad relacional, abierta, y transitoria regida por el espacio y el tiempo en que vive la población involucrada, porque absorbe, resiste y se recupera como forma de preservación y coadyuvante en el equilibrio y (o) afrontamiento de los efectos del cambio climático como parte de la estabilidad de la condición humana en regiones que son amenazadas por determinados fenómenos naturales extremos de origen climático.

La capacidad de adaptación a manera de cualidad de la resiliencia como medida de la estabilidad de cualquier sistema, es la que puede ayudar a contrarrestar los efectos de eventos externos porque intrínsecamente se muestra como coadyuvante desde perspectivas socio-ambientales a la mejora de la estabilidad de la condición humana en regiones que son amenazadas por dichas circunstancias.

Desde esta perspectiva a través de la adaptación planificada de la resiliencia que considera lo territorial, lo espacial y los sistemas construidos, busca generar condiciones que favorezcan y ayuden a la preservación, rescate y habitabilidad de las viviendas tradicionales de los pueblos originarios en Bolivia.

\section{Referencias bibliográficas}

Aguilar, J. (2015). Variabilidad espacio-temporal de la temperatura de la superficie del suelo en el Perú y su relación con los eventos extremos, mediante imágenes MODIS/Terra durante el periodo 2000-2012 (Tesis de Magister) Universidad Nacional Mayor de San Marcos, Lima, Perú. https://cybertesis.unmsm.edu.pe/bitstream/handle/20.500.12672/9989/Aguilar_lj.pdf?sequence=3\&isAll owed $=\mathrm{y}$

Altieri, M. et. al. (2015). Agroecology and the design of climate change-resilient farming systems. INRA and Springer, 35, 869-890. https://link.springer.com/article/10.1007/s13593-015-0285-2

Atencia, D. J.; Plaza, M. T. y Hernández, H. E. (2020). Resiliencia, burnout y fracaso académico en estudiantes de Ingeniería de la Universidad de Córdoba, Colombia. Revista Espacios. 41(11), 23. Recuperado de https://www.revistaespacios.com/a20v41n11/20411123.html\#iden

Ávila, B. y Gonzáles, G. (julio-diciembre, 2015). Sociedades resilientes: criterios para estrategias educativas encaminadas a la reducción de riesgo de desastres. Revista Interamericana de Educación de Adultos, 37(2), 26-46. https://www.redalyc.org/pdf/4575/457544924003.pdf

Bahadur, A. et. al. (2010). The resilience renaissance? Unpacking of resilience for tackling climate change and disasters. 
https://www.researchgate.net/publication/275831843_The_Resilience_Renaissance_Unpacking_of_Resili ence_for_Tackling_Climate_Change_and_Disasters_Brighton_IDS_SCR_Working_Paper

Barabas, A. (2014). Multiculturalismo, pluralismo cultural y interculturalidad en el contexto de América Latina: la presencia de los pueblos originarios. Configurações, 14, 11-24.

https://journals.openedition.org/configuracoes/2219

Baró, J. y Monroy, F. (2018). Enfrentando los riesgos

socionaturales.http://www.educacionchiapas.gob.mx/pcivil/2019/material/Enfrentando\%20los\%20riesgos \%20socionaturales.pdf

Barton, J. (2009). Medio ambiente urbano Adaptación al cambio climático en la planificación de ciudadesregiones. Revista de Geografía Norte Grande, 43, 5-30. https://scielo.conicyt.cl/pdf/rgeong/n43/art01.pdf

Borgatti, S., et. al. 2002. UCINET for Windows: Software for social network analysis. https://www.researchgate.net/publication/216636663_UCINET_for_Windows_Software_for_social_netw ork_analysis

Camarero, L. y Del Pino, J. (mayo-agosto, 2012). Cambios en las estructuras de los hogares rurales. Revista Internacional de Sociología, 72(2), 377-401. http://doi:10.3989/ris.2012.12.27

Centro Internacional para la Investigación del Fenómeno de El Niño ([CIIFEN], 2010). Centro Internacional para la Investigación del Fenómeno de El Niño.

http://www.ciifen.org/index.php?option=com_content\&view=category\&layout=blog\&id=84\&ltemid=336 \&lang=es

Chacón, J. (2008). Los conceptos actuales de susceptibilidad, peligrosidad y riesgo, en la prevención de movimientos de ladera, con ejemplos de aplicaciones prácticas.

https://www.researchgate.net/publication/258856593_Los_conceptos_actuales_de_susceptibilidad_pelig rosidad_y_riesgo_en_la_prevencion_de_movimientos_de_ladera_con_ejemplos_de_aplicaciones_practic as

Clerc, J. y Díaz, M. (2016). Un marco para estimar los costos incrementales del cambio climático en infraestructura. https://publications.iadb.org/es/publicacion/15541/un-marco-para-estimar-los-costosincrementales-del-cambio-climatico-en

Del cura, F. y Quintero, E. (2016). A4 - 98 Aproximaciones a la medición de la resiliencia en comunidades rurales del Estado Mérida, Venezuela, ante escenarios de cambio climático.

http://sedici.unlp.edu.ar/bitstream/handle/10915/58395/Documento_completo.pdfPDFA.pdf?sequence=1\&isAllowed $=y$

Doring, T. et. al. (2013). Resilience as a universal cretrion of health. J Sci Food Agric., 95, 455-465. https://onlinelibrary.wiley.com/doi/epdf/10.1002/jsfa.6539

Fisichelli, N. et. al. (2016). Is 'Resilience' Maladaptive? Towards an Accurate Lexicon for Climate Change Adaptation. Environmental Management, 57, 753-758. https://link.springer.com/article/10.1007/s00267015-0650-6

Gonzáles, E., et. al.(2019). Nuevos desafíos para la educación ambiental: la vulnerabilidad y la resiliencia social ante el cambio climático. Revista Cuadernos de Investigación UNED, 11(1), S71-S77. https://www.scielo.sa.cr/pdf/cinn/v11n1/1659-4266-cinn-11-01-71.pdf 
González, G. y Maldonado, L. (2017). Amenazas y riesgos climáticos en poblaciones vulnerables. El papel de la educación en la resiliencia comunitaria. Revista Teor. Educ., 29, 273-294. Recuperado de https://gredos.usal.es/bitstream/handle/10366/133990/Amenazas_y_riesgos_climaticos_en_poblaci.pdf? sequence=1\&isAllowed=y

Grupo de las Naciones Unidas para el Desarrollo ([UNDG], 2009). Integrando la reducción del riesgo de desastres en ela CCA y el Manud.

https://www.unisdr.org/files/10760_undgdrrguidancenotespanish28lowreso.pdf

Guarino, G. y Pironto, A. (marzo-junio, 2019). Patrimonio biocultural y resiliencia en los pueblos indígenas del Chaco, Argentina. Revista INVENTIO,35, 33-43. http://inventio.uaem.mx/index.php/inventio/article/view/694/0

Hardy, V., et. al. (abril-junio, 2019). Aprendizaje y resiliencia en la gestión local de riesgos de desastres. Revista Luz, 2, 42-52. https://luz.uho.edu.cu/index.php/luz/article/view/965

Holling, S. (1973). Resilience and stability of ecological systems. Annual Review of Ecology and Systematics, 4(1) , 1-23. https://www.annualreviews.org/doi/abs/10.1146/annurev.es.04.110173.000245

Intergovernmental Panel on Climate Change ([IPCC], 2014). Cambio climático 2014: Impactos, adaptación y vulnerabilidad - Resumen para responsables de políticas. Contribución del Grupo de trabajo II al Quinto Informe de Evaluación del Grupo Intergubernamental de Expertos sobre el Cambio Climático.https://www.ipcc.ch/site/assets/uploads/2018/03/ar5_wgll_spm_es-1.pdf

Jiménez, M. (2013). ¿Resiliencia ante el cambio climático?.http://www.ccpy.gob.mx/ecologiahumana/documentos/Resiliencia-ante-el-cambio-climatico.pdf

Kenneth, A., et. al. (2015). Operationalizing resilience for adaptive coral reef management under global environmental change. Revista Global Change Biology, 21, 48-61. https://core.ac.uk/download/pdf/51090649.pdf

Lara, R. y Vides-Almonacid, R. (2014). Sabiduría y Adaptación: El Valor del Conocimiento Tradicional en la Adaptación al Cambio Climático en América del Sur. UICN: Quito, Ecuador. https://portals.iucn.org/library/sites/library/files/documents/2014-001.pdf

Leichenko, R. (2011). Climate Change and Urban Resilience. https://www.researchgate.net/publication/235981318_Climate_Change_and_Urban_Resilience

Levinea, N., et. al. (2016). Ecosystem heterogeneity determines the ecological resilience of the Amazon to climate change. Revista PNAS, 113(3), 793-797. https://www.pnas.org/content/113/3/793

Magrin, G. (2015). Adaptación al cambio climático en América Latina y el Caribe.https://www.cepal.org/es/publicaciones/39842-adaptacion-al-cambio-climatico-america-latinacaribe

Mateo, J. (2004). Planificación y gestión ambiental, La Habana, Cuba: Editorial de la Universidad de La Habana.

Ministerio de Ambiente y Desarrollo Sostenible de Colombia. ([MADSC], 2014). Protocolo para La Incorporación de la Gestión del Riesgo en los Planes de Ordenación y Manejo de Cuencas Hidrográficas. https://www.minambiente.gov.co/images/GestionIntegraldelRecursoHidrico/pdf/Plan-de-ordenamientodel-Recurso-Hidrico/PROTOCOLO_INCORP._GESTION_DEL_RIESGO_-_POMCA.pdf 
Narváez, L. et. al. (2009). La gestión del Riesgo de Desastres. Un enfoque basado en procesos.http://www.comunidadandina.org/predecan/doc/libros/PROCESOS_ok.pdf

Oficina de las Naciones Unidas para la Reducción del Riesgo de Desastres ([UNISDR], 2009). Terminología sobre la reducción del riesgo de desastre.http://www.unisdr.org/files/7817_UNISDRTerminology Spanish.pdf

Ojeda, D. D. y Steffens, E. J. (2017). Resiliencia y su influencia en vendedores de empresas multinivel colombianas. Revista Espacios. 38(61), 23. Recuperado de https://www.revistaespacios.com/a17v38n61/17386123.html\#iden2

Organización de las Naciones Unidas ([ONU], 2015). Adaptación al cambio climático en América Latina y el Caribe.https://www.cepal.org/es/publicaciones/39842-adaptacion-al-cambio-climatico-america-latinacaribe

Papa, R. et. al. (2015). Cities, energy and cliimate change. Revista Tema Journal of Land Use, Mobility and Environment, 8(1), 19-49. http://eprints.bice.rm.cnr.it/11247/1/03_2883-14032-1PB\%20papa\%20galderisi.pdf

Peredo, S. y Vela, M. (2016). Determinación de los niveles de resiliencia/vulnerabilidad en iniciativas de agroecología urbana en el suroeste Andaluz. Revista IDESIA,34(2) ,5-13. https://scielo.conicyt.cl/pdf/idesia/v34n2/aop0316.pdf

Picket, S. et. al. (2014). Ecological resilience and resilient cities.https://www.researchgate.net/publication/263145263_Ecological_resilience_and_resilient_cities

Pradilla, G. (2016). Análisis ambiental de las prácticas campesinas de resiliencia a la variabilidad y el cambio climático en fincas ecológicas del altiplano Cundiboyacense (Tesis de Magister) Universidad Nacional de Colombia, Bogotá D. C., Colombia. ttp://bdigital.unal.edu.co/52717/1/pradilla.2016.resiliencia.pdf

Programa de Naciones Unidas para el Desarrollo ([PNUD], 2011). Tras las huellas del cambio climático en Bolivia. https://idl-bnc-idrc.dspacedirect.org/bitstream/handle/10625/59246/59401.pdf?sequence=1

Quevedo, A. (abril-junio, 2017). Nuevas formas de enseñanza en gestión ambiental para la reducción de la vulnerabilidad y la adaptación al cambio climático en Bolivia. Revista CIEG,28, 213-225. http://www.grupocieg.org/archivos_revista/Ed.\%2028(213-225)-Quevedo\%20Quispe\%20Winstonabril2017_articulo_id312.pdf

Quinlan, A. et. al. (2016). Measuring and assessing resilience: broadening understanding through multiple disciplinary perspectives. Journal of Applied Ecology, 53, 677-687. https://besjournals.onlinelibrary.wiley.com/doi/full/10.1111/1365-2664.12550

Real Academia Española ([RAE], 2019). Adaptación. Recuperado de https://dle.rae.es/resilien Real Academia Española ([RAE], 2020a). Resiliencia. https://dle.rae.es/resiliencia?m=form Real Academia Española ([RAE], 2020b). Función.https://dle.rae.es/funci\%C3\%B3n Real Academia Española ([RAE], 2020c). Absorber. https://dle.rae.es/absorber?m=form Real Academia Española ([RAE], 2020d). Resistir. https://dle.rae.es/resistir?m=form Real Academia Española ([RAE], 2020e). Recuperar. https://dle.rae.es/recuperar Real Academia Española ([RAE], 2020f). Preservación.https://dle.rae.es/preservaci\%C3\%B3n?m=form 
Rebotier, J., et. al. (febrero-mayo, 2013). Las paradojas de la resiliencia: miradas cruzadas entre Colombia y Francia. Revista Territorios,28, 127-145. https://www.redalyc.org/pdf/357/35728173006.pdf

Rivero, E. (2017). Actitudes resilientes ante el cambio climático en Achocalla. Revista Desafíos Educativos, 17, 83-103. http://www.scielo.org.bo/pdf/rip/n17/n17_a06.pdf

Rodríguez, Y. (2018). Potenciar la resiliencia de las ciudades y sus territorios de pertenencia en el marco de los acuerdos sobre cambio climático y de la Nueva Agenda Urbana. https://repositorio.cepal.org/bitstream/handle/11362/44218/S1800995_es.pdf?sequence=1\&isAllowed=y

Rojas, O. y Martínez, C. (2011). Riesgos naturales: evolución y modelos conceptuales. Revista Universitaria de Geografía, 20, 83-116. http://bibliotecadigital.uns.edu.ar/pdf/reuge/v20n1/v20n1a05.pdf

Romero, O. M.; Jaramillo, M. F.; Aguirre, M.C. y Ruiz, J.A. (2017). Resiliencia y percepción de oportunidades de negocio de los estudiantes de la Universidad Técnica de Machala, Ecuador. Revista Espacios. 38(50), 23. Recuperado de https://www.revistaespacios.com/a17v38n50/17385023.html\#iden

Sánchez, P. et. al. (2016). La noción de resiliencia en el análisis de las dinámicas territoriales rurales: Una aproximación al concepto mediante un enfoque territorial. Revista Cuadernos de Desarrollo Rural, 13(77), 93-116. http://www.scielo.org.co/pdf/cudr/v13n77/0122-1450-cudr-13-77-00093.pdf

Sandoval, J. (2020). Vulnerabilidad-resiliencia ante el proceso de riesgo-desastre: Un análisis desde la ecología política. Revista Polis, 56. http://journals.openedition.org/polis/19313

Sepúlveda, J. (2015). Acciones de adaptación y mitigación al cambio climático en la planificación de la ciudad de Medellín (Tesis de magister) Universidad Nacional de Colombia, Medellín, Colombia.

https://repositorio.unal.edu.co/bitstream/handle/unal/55419/43998063.2015.pdf?sequence=1\&isAllowe $\mathrm{d}=\mathrm{y}$

Shaikh, K. y Saidul, M. (2016). Community Capitals as Community Resilience to Climate Change: Conceptual Connections. Revista Int J Environ Res PublicHealth, 13(12), 1211. https://pubmed.ncbi.nlm.nih.gov/27929448/

Skerk, C., et. al. (2014). La infraestructura en el desarrollo de América Latina. Tendencias y novedades de la infraestructura en la región. https://scioteca.caf.com/handle/123456789/750

Soldano, A. (2015). Conceptos sobre Riesgo. http://www.rimd.org/advf/documentos/4921a360071e58.79575639.pdf

Stanley, C. (1973). Resilience and stability of ecological systems. Annual Review of Ecology and Systematics, 4, 1-23. https://www.annualreviews.org/doi/abs/10.1146/annurev.es.04.110173.000245

Timpane, B. et. al. (2017). A systematic review of ecological attributes that confer resilience to climate changein environmental restoration. PLOS ONE, 12(3):e0173812. https://doi.org/10.1371/journal.pone.0173812

UN-Habitat (2016). Resiliencia Urbana. https://es.unhabitat.org/resiliencia/

Uriarte, J. (2010). La resiliencia comunitaria en situaciones catastróficas y de emergencia. Revista INFAD, 1(1), 687-693. https://www.redalyc.org/pdf/3498/349832324073.pdf

Vargas J. y Paneque, P., 2016. Metodología para el análisis de las causas de la vulnerabilidad al riesgo de sequía a escala de demarcación 
hidrográfica.https://www.researchgate.net/publication/311626665_Metodologia_para_el_analisis_de_las _causas_de_la_vulnerabilidad_al_riesgo_de_sequia_a_escala_de_Demarcacion_Hidrografica

Zúñiga, I. (enero-junio, 2017). Nociones sobre resiliencia urbana y territorial. Revista del ordenamiento territorial y el urbanismo, 24, 43-48.

http://www.ipf.gob.cu/sites/default/files/revista/Nociones\%20sobre\%20resiliencia...pdf

Zúñiga, I. (enero-abril, 2018). Resiliencia urbana ante inundaciones por intensas lluvias en contribución al desarrollo urbano equilibrado. Revista Arquitectura y Urbanismo, 39(1), 39-50.

http://rau.cujae.edu.cu/index.php/revistaau/article/view/441/413

Zúñiga, et. al. (2020). Capacidad de carga y resiliencia de los sistemas socio-ambientales desde un enfoque sostenible. Revista Monteverdia, 3(2), 51-56. https://revistas.reduc.edu.cu/index.php/monteverdia (coautor de esta investigación).

Esta obra está bajo una Licencia Creative Commons

Attribución-NoCommercial 4.0 International

(c) EY-NC 